\title{
S-C-6. A Frontal Appreach to the Third Ventricle Tumors
}

\author{
Masashichi KawaNo \\ Second Department of Surgery, Nagasaki University School of Medicine
}

With only exception of colloid cyst which situates at the feramen of Monroe, tumors in the third ventricle have been attacked surgically through the cerpus callesum following occipital or parietal craniotomy. The serious disadvantage of this appreach is the presence of the Galen's great cerebral vein and its tributaries which run behind and over the third ventricle. Technical difficulty increases when the mass in the third ventricle is a large one. For such large mass the frontal approach has a definite advantage. One reaches to the right anterior horn of the lateral ventricle through a vertical incision of the right frontal lobe. From this opening one can visualize the third ventricle mass either through the feramen of Menroe or through the thinned wall of the third ventricle, which can be opened without leaving serious damage to the hypothalamus or thalamus. The tumor mass can be extirpated with the help of fingers and forceps with good view of the whole mass and the adjoining structure. There is minimal chance of bleeding from the dangerous veins in the vault and back of the enlarged third ventricle.

So far, this technic was applied to only 3 cases. The first case was a 7 -month-old boy with an enormous mass of $210 \mathrm{Gms}$ with the shape of acardius acephalus which was successfully removed en mass and no neurological deficit was left in the follow-up for 15 months. The mass contained both lower extremities, one upper extremity, abdominal aorta, portions of gastrointestinal canal and brain. Inclusion fetus or fetal inclusion would be the proper name for this type of congenital mass. The second case was a 8-year-old boy with pinealoteratoma of $58 \mathrm{Gms}$. which was removed successfully, but the boy died of infection through the ventricular drainage 70 days after operation. The third case was a 9-year-old girl who has had obstructive hydrocephalus which was treated with ventriculo-atrial shunt. The mass in the third ventricle was subtotally removed but the attachment to the base of the skull was tight and extensive. The girl died of pneumenia and gastric hemorrhage 7 days after operation without regaining consciousness. Histologically the tumor was craniopharyngioma, although no calcification was seen on $\mathrm{X}$-ray films.

From these small series of experience we learn that the frontal approach is indicated in large third ventricle tumer mass including pinealoma, but 
it is not the ideal appreach if the tumor arises from the base of the cerebrum or the skull, because the damage to the hypothalamus then can be serious.

It requires further clinical experience and evaluation before this approach be established as a standard technic for a certain group of third ventricle tumors.

\title{
Symposium D. Congenital Anomalies
}

\author{
Chairman: Prof. Takao Mitsuno \\ Dept. of Surgery, Kobe University
}

\section{S-D-1. Surgery of Developmental Anomalies due to Dysraphic State}

\author{
Hiroshi FukaI \\ Brain Research Institute, Niigata University School of Medicine
}

Among the congenital malformations of the central nervous system, it is well known that a dysraphic state plays the most important role. There are two theories on the pathogenesis of the dysraphic state: one is the araphic of dysraphic theory, namely, a failure of closure, rather than rapture, of the neural tube, another is the hydrodynamic theory, namely, overdistension of the neural tube, i.e. hydroencephalomyelic state.

In general, the concept of the dysraphic state of the central nervous system indicates the congenital or developmental anomalies due to impairment of formation of the neural tube in the early embryonal life. Then, the dysraphic states includes various pathological conditions, but a few of which have indications for surgical treatment, because most of them are uncurable by surgical intervention.

Operation of the developmental anomalies of the central nervous system are performed to intend (1) improvement of manifested neurological dysfunction, (2) reconstruction of the abnormal anatomical structures, (3) prevention of neurological deficit and/or complication which will ensue, and (4) cosmetic effect.

The following developmental anomalies are discussed from a neurosur- 